wurde eine weitere positive randomisierte Phase-III-Studie bei EGFR-Mutation bei asiatischen Patienten präsentiert (\#8016). Für Patienten mit Eml4-alkAlteration wurde eine zweite sehr wirksame Substanz, das LDK378, vorgestellt, das in der Primärtherapie von rezidivierten Patienten mit Alk-Rearrangement um $60 \%$ objektive Remissionen zeigt, aber auch bei mit Crizotinib-vor- behandelten Patienten praktisch vergleichbare Effektivität (60\% OR) bestätigt (\#TPS8119). Weitere wichtige Treiberalterationen mit Möglichkeit der Intervention dürften die BRAF-V600EMutation (\#8009), das RET-Rearrangement (\#8024) und ein NTRK-Rearrangement (\#8023) sein. Die Second-Line-Behandlung könnte bei Adenokarzinomen durch das Nitedanib relevant verbessert werden (LUME-1) bei einem medianen Overall Survival-Benefit von mehr als zwei Monaten (\#LBA8011).

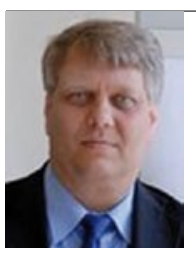

\title{
Magenkarzinom und Pankreaskarzinom im Fokus
}

Die Rolle biologischer Zielstrukturen des fortgeschrittenen Magenkarzinoms wird weiter erforscht. HER2-Überexpression erweist sich in der randomisierten EXPAND-Studie, die Patienten mit HER2-positiven und HER2-negativen Tumoren in eine Behandlung mit Chemotherapie +/- Cetuximab randomisierte, als günstiger Prognoseparameter unter Therapie. Patienten mit HER2-positiven Magenkarzinomen sprechen besser auf Chemotherapie an und leben länger (\#4021). Hingegen ist der gegen HER2 und EGFR gerichtete Kinaseinhibitor Lapatinib bei HER2-positiven Magenkarzinomen in der randomisierten LOGIC-Studie nicht ausreichend wirksam (\#4001).

Die Second-Line-Therapie des Magenkarzinoms wird konkreter. In der randomisierten Cougar-02-Studie wurde eine signifikante Verlängerung des Überlebens von 3,6 auf 5,2 Monate mit Docetaxel versus alleinige Symptomkontrolle erreicht. Insbesondere die Schmerzkontrolle ist mit Chemotherapie verbessert. Der allgemeine Gesundheitszustand nach EORTC QLQ C-30 war in beiden Gruppen gleich. Der Benefit einer Zweitlinientherapie kommt vor allem bei Patienten in gutem Performance Status $(\mathrm{ECOG}=0)$ und nach längerem progressionsfreiem Intervall nach erster Linie (> 3 Monate) zum Tragen (\#4003).

Die Systemtherapie des Pankreaskarzinoms entwickelt sich weiter. Nach $\mathrm{Pu}$ blikation der Ergebnisse mit FOLFIRINOX erweist sich nun auch die Kombination von Albumin-gebundenem Paclitaxel mit Gemcitabin einer GemcitabinMonotherapie als überlegen. Der Unter- schied im Überleben beträgt 6,7 versus 8,5 Monate (\#4005).

Als wirkungslos zeigt sich in der randomisierten LAP-07-Studie dagegen die Hinzunahme einer Radiotherapie zur systemischen Chemotherapie bei lokal fortgeschrittenem, nicht fernmetastasiertem Pankreaskarzinom. Somit bleibt der Stellenwert einer Radiotherapie des Pankreaskarzinoms bis auf weiteres undefiniert (\#4003).

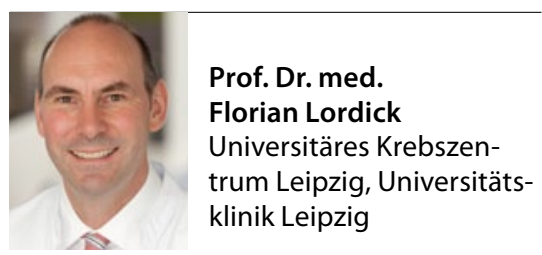

Ticker +++ Ticker +++ Ticker +++ Ticker +++ Ticker +++ Ticker +++ Ticker +++ Ticker +++ Ticker +++ Ticker +++ Ticker +++ Ticker +++

Zweitlinientherapie geprüft: 255 vorbehandelte Patienten mit Adenokarzinomen der Lunge im Stadium IIIB/IV erhielten entweder eine Docetaxel-Monotherapie, d.h. einen derzeitigen Standard beim NSCLC, oder die Kombination aus HSP90-Inhibitor und Taxan [Ramalingam $S$ et al. J Clin Oncol. 2013;31(suppl;abstr. CRA8007)].

Das progressionsfreie Überleben als primärer Endpunkt wurde durch das Zweierregime um 1,3 Monate verlängert, das Progressionsrisiko um $16 \%$ gesenkt (4,5 vs. 3,2 Monate; HR 0,84; $p=0,038)$. Auch beim Gesamtüberleben gab es mit 9,8 Monaten einen Benefit zugunsten der Kombination (Docetaxel: 7,4 Monate). Besonders profitierten $\mathrm{Pa}$ tienten von der Ganetespib-basierten Therapie, bei denen das fortgeschrittene NSCLC vor mehr als sechs Monaten diagnostiziert worden war: In diesem Subkollektiv $(n=177)$ wurde das progressionsfreie Überleben um zwei Monate, das Gesamtüberleben um mehr als vier Monate verbessert (5,4 vs. 3,4 Monate bzw. 10,7 vs. 6,4 Monate). Der Überlebensvorteil durch
Ganetespib korrelierte jedoch nicht mit dem KRAS- oder EGFRMutationsstatus.

(arn)

Fatigue bei Lungenkrebs: Modafinil wenig wirksam

Patienten mit lokal fortgeschrittenem oder metastasiertem Lungenkarzinom, die unter Fatigue leiden, profitieren nicht von der Behandlung mit dem psychostimulatorisch wirksamen Medikament Modafinil [Fife K et al. J Clin Oncol. 2013;31(suppl;abstr. 9503)]. In der englischen Untersuchung, in der die Substanz in einer Do- sierung von $100 \mathrm{mg} /$ Tag über 14 Tage und nochmals $200 \mathrm{mg} / \mathrm{Tag}$ über weitere 14 Tage mit Placebo verglichen wurde. Von 208 Patienten beendeten 160 Patienten die Therapie. Gemessen mithilfe der FACIT (Functional Assessment of Chronic Illness Therapy)Fatigue-Skala erreichten die mit Modafinil behandelten Patienten eine Verbesserung um 5,28 Punkte, die mit Placebo behandelten $5,11.47 \%$ der Patienten im Modafinil-Arm und $23 \%$ im Placebo-Arm sagten aus, dass die Behandlung nicht geholfen habe. 